\title{
REVISÃO E PLANEJAMENTO DE EXPERIMENTOS COM AÇO E ESCÓRIA EM FORNO DE LABORATÓRIO PARA O ESTUDO DE INCLUSÕES*
}

Pedro Cunha Alves Vinicius Cardoso da Rocha ${ }^{2}$ Julio Aníbal Morales Pereira ${ }^{3}$ Wagner Viana Bielefeldt ${ }^{4}$ Antônio Cezar Faria Vilela ${ }^{5}$

\section{Resumo}

A cada ano a demanda por aços limpos aumenta, tornando o controle de inclusões cada vez mais importante. Durante o refino secundário de aços, ocorrem a eliminação de impurezas, ajuste da composição química e ajuste da temperatura do aço líquido. A remoção de inclusões não-metálicas geralmente ocorre nessa etapa, sendo realizada pela escória. Neste trabalho, foi definido um planejamento de experimentos para que numa segunda etapa, os conhecimentos obtidos possam ser comparados e transferidos para a prática industrial. Inicialmente, foi feita uma revisão da literatura sobre experimentos realizados em fornos de laboratório com amostras de aços e escórias focados na remoção de inclusões. Depois de analisados diferentes arranjos experimentais propostos, alguns trabalhos selecionados foram analisados com respeito às inclusões e às escórias presentes. Finalmente, no planejamento proposto, definiu-se que os experimentos serão realizados em forno resistivo com passagem de argônio. As amostras serão fundidas em cadinhos de $\mathrm{MgO}$ na temperatura de $1600^{\circ} \mathrm{C}$ e, após 90 minutos nesta temperatura, as mesmas serão resfriadas em água. Alguns parâmetros que poderão ser estudados são a população de inclusões, viscosidade efetiva da escória, teor de oxigênio total e composição de inclusões ao longo do tempo.

Palavras-chave: Refino secundário; Limpeza de aços; Forno resistivo; Inclusões.

\section{REVIEW AND PLANNING OF EXPERIMENTS WITH STEEL AND SLAG IN LABORATORY FURNACE TO THE STUDY OF INCLUSIONS}

\begin{abstract}
Every year the demand for clean steels increases, making inclusion control each time more important. During the steel secondary refining, occurs the elimination of impurities, adjustment of the chemical composition and adjustment of the temperature of the liquid steel. The removal of non-metallic inclusions usually occurs in this step, being performed by the slag. In this work, a planning of experiments was defined so that in a second stage, the obtained knowledge can be compared and transferred to the industrial practice. Initially, a review of the literature on experiments carried out in laboratory furnaces with samples with steel and slag was carried out in studies focused on the removal of inclusions. After analyzing different proposed experimental arrangements, some selected works were analyzed with respect to the inclusions and the present slag. Finally, in the proposed planning, the experiments will be carried out in a resistive furnace with argon passage. The samples will be melted in $\mathrm{MgO}$ crucibles at $1600^{\circ} \mathrm{C}$ and, after 90 minutes at this temperature, they will be cooled in water. Some parameters that could be study are the population of inclusions, effective viscosity of the slag, total oxygen content and composition of inclusions over time.
\end{abstract}

Keywords: Secondary refining; Steel cleanliness; Resistive furnace; Inclusions.

\footnotetext{
Eng. Metalúrgico, mestrando, Laboratório de Siderurgia (LaSid), Depto. de Metalurgia, PPGE3M/UFRGS, Porto Alegre, RS, Brasil. MSc Eng. Metalúrgico, doutorando, LaSid, Depto. de Metalurgia, PPGE3M/UFRGS, Porto Alegre, RS, Brasil.

Dr Eng. Metalúrgico, pós-doutorando, LaSid, Depto. de Metalurgia, PPGE3M/UFRGS, Porto Alegre, RS, Brasil.

Professor, Dr., LaSid, Depto. de Metalurgia, PPGE3M/UFRGS, Porto Alegre, RS, Brasil

Professor, Dr.-Ing., LaSid, Depto. de Metalurgia, PPGE3M/UFRGS, Porto Alegre, RS, Brasil.
} 


\section{INTRODUÇÃO}

Durante a fabricação do aço, o processo de refino secundário é uma das etapas mais importantes para se obter um produto de alta qualidade. Durante a etapa de refino, ocorrem a eliminação de impurezas, ajuste da composição química e ajuste da temperatura do aço líquido. Neste processo, são usualmente utilizados dois equipamentos: o forno-panela e o desgaseificador a vácuo. Sendo o segundo, parte importante na fabricação de aços especiais [1].

Escórias de refino secundário são compostas de óxidos básicos e ácidos. Entre suas principais funções estão: absorver impurezas [2,3], evitar a reoxidação [4] e proteger termicamente o aço líquido [4]. Dois tipos de sistemas de escórias são comumente utilizados. $\mathrm{O}$ sistema $\mathrm{CaO}-\mathrm{SiO}_{2}-\mathrm{Al}_{2} \mathrm{O}_{3}-\mathrm{MgO}\left(-\mathrm{CaF}_{2}\right)$ e o sistema $\mathrm{CaO}-\mathrm{SiO}_{2}-\mathrm{Al}_{2} \mathrm{O}_{3}-\mathrm{MgO}$ $[5,6]$.

As escórias com adição de fluorita $\left(\mathrm{CaF}_{2}\right)$, normalmente, apresentam valores entre 10 e $20 \%$ em massa de $\mathrm{Al}_{2} \mathrm{O}_{3}$ e no máximo $12 \%$ de $\mathrm{CaF}_{2}$. Para valores mais altos de $\mathrm{Al}_{2} \mathrm{O}_{3}(\sim 25 \%)$, a fluorita é adicionada em valores de até $5 \%$ em massa, agindo como fluxante inicial [6]. As escórias com fluorita apresentam alta basicidade e possuem como características: boa desoxidação, boa dessulfuração e boa capacidade para remover inclusões [5-7]. A adição de fluorita é benéfica para algumas propriedades da escória, como por exemplo a redução da viscosidade $[3,6]$, porém pode causar problemas ambientais e de desgaste do refratário, exigindo maior atenção quando utilizada $[5,7]$.

As escórias sem $\mathrm{CaF}_{2}$, normalmente, apresentam altos valores de $\mathrm{Al}_{2} \mathrm{O}_{3}$ (entre 20 e $30 \%$ em massa) e valores de $\mathrm{SiO}_{2}$ abaixo de $20 \%$. Nessa configuração, é possível atingir melhores resultados para a solubilidade do $\mathrm{CaO}$ [6]. Essas escórias possuem baixo ponto de fusão, baixa viscosidade e podem melhorar a limpeza do aço $[5,8]$.

Um dos principais parâmetros para medir a limpeza do aço é o controle de inclusões não-metálicas. Entre esses parâmetros se destacam: composição, tamanho, morfologia e distribuição [9]. A demanda por aços limpos aumenta a cada ano, assim o controle de inclusões se torna cada vez mais importante [10]. A análise do teor de oxigênio total é uma das formas mais utilizadas para avaliar a limpeza do aço. Este valor inclui a quantidade de oxigênio dissolvido no aço mais a quantidade de oxigênio presente nas inclusões não-metálicas [11,12]. Avanços nos processos de refino levaram a valores muito baixos nos últimos anos, acarretando no decréscimo do número de inclusões [11]. Dependendo da aplicação, o controle de inclusões deve ser feito de maneira diferente, desse modo, diferentes aplicações requerem diferentes práticas para controle das inclusões [9].

As inclusões encontradas em aços se dividem em dois tipos [13]:

a) Inclusões endógenas: formadas nas reações de refino ou no resfriamento do aço (por precipitação). Estão relacionadas com as reações dos elementos adicionados que possuem alta afinidade com oxigênio, enxofre e nitrogênio [13].

b) Inclusões exógenas: geralmente formadas por quebra ou desgaste do refratário, arraste de escória, pó de molde ou pó de cobertura do distribuidor, entre outros fatores. Possuem formato irregular e ocorrem esporadicamente [13].

Os efeitos das inclusões no desempenho e nas propriedades dos aços são bem conhecidos. Inclusões de óxidos e de sulfetos, em geral, têm efeito negativo nas propriedades mecânicas como resistência à fadiga, dureza, ductilidade e conformabilidade. Um controle adequado das inclusões pode diminuir seus efeitos negativos e até obter efeitos positivos em algumas propriedades [11]. 
As inclusões são removidas geralmente por absorção pela escória. Na produção de aço, a remoção ocorre no refino secundário, distribuidor e lingotamento contínuo. $O$ processo de remoção é realizado em três passos: (i) flotação, (ii) separação, (iii) dissolução [14]. O primeiro passo envolve o transporte da inclusão até a interface aço/escória. $\mathrm{Na}$ interface estão presentes tensões superficiais que precisam ser atingidas para realizar a separação da inclusão do aço líquido e sua estabilização na interface aço/escória. Por último, a inclusão é considerada removida quando a mesma é totalmente incorporada à escória [2,14].

Escórias de refino têm grande efeito na limpeza do aço, proporcionando um decréscimo no teor de oxigênio total devido ao seu poder de desoxidação, levando a diminuição do número de inclusões [15]. Outros fatores que podem ser modificados com as escórias de refino são o formato, composição e o ponto de fusão das inclusões. Diversos estudos [2,3,8,11,14,16,17] já foram realizados com objetivo de avaliar a influência da composição química das escórias na limpeza inclusionária dos aços.

Visando um melhor entendimento e posterior melhoria de processo industrial, uma boa alternativa é a realização de experimentos com aço e escória em forno de laboratório. Nesses experimentos, são possíveis estudar as diferentes características da escória, tais como sua basicidade binária, teor de alumina $\left(\mathrm{Al}_{2} \mathrm{O}_{3}\right)$, teor de sílica $\left(\mathrm{SiO}_{2}\right)$ e teor de $\mathrm{MgO}$, bem como seus efeitos no controle de inclusões. Uma boa estratégia é adotar o conhecimento inicial em laboratório, junto a testes na indústria a fim de validar os resultados obtidos [15,18-21].

O objetivo principal deste trabalho é elaborar um arranjo experimental, a partir de uma revisão bibliográfica, para a realização de experimentos em forno de laboratório que possibilitem estudar fenômenos do refino secundário, visando a remoção e modificação de inclusões. Os objetivos secundários são: apresentar os parâmetros que serão fixados durante os experimentos, introduzir alguns estudos já realizados com parâmetros semelhantes, mostrando exemplos do que pode ser pesquisado, e concluir sobre alguns efeitos que podem ocorrer devido às propriedades da escória.

\section{MATERIAIS E MÉTODOS}

\subsection{Definição de Parâmetros Experimentais}

Para definir um arranjo para testes de laboratório com escória em operações do refino secundário, foram definidos três passos iniciais conforme a figura 1.

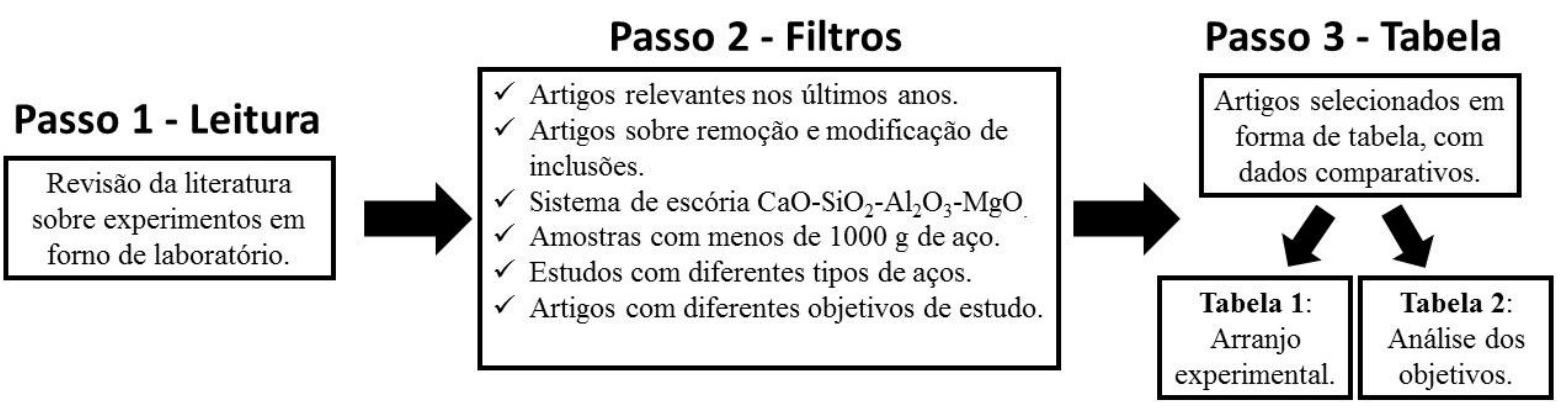

Figura 1. Fluxograma para formação da tabela com dados experimentais comparativos.

O primeiro passo foi focado na leitura de artigos selecionados a partir da revisão da literatura especializada. No segundo passo foram aplicados filtros e critérios. Dentro do segundo passo foram selecionados trabalhos realizados com aços especiais baixa 
liga e aços inoxidáveis. Com isso, buscou-se verificar as diferentes práticas, dependendo do tipo de aço estudado. Na definição dos filtros, se identificou que poucos experimentos utilizavam fluorita na composição da escória, com isso optou-se por deixar este fator para um segundo momento. Para este estudo, será utilizado somente 0 sistema de escória $\mathrm{CaO}-\mathrm{SiO}_{2}-\mathrm{Al}_{2} \mathrm{O}_{3}-\mathrm{MgO}$ para se adquirir prática laboratorial neste tipo de experimento. Estudos desenvolvidos com mais de $1000 \mathrm{~g}$ de aço também foram excluídos devido ao fator limitante do forno que será usado. Da analise da tabela 1, resultará a proposta do arranjo experimental.

\subsection{Selecionar Parâmetros de Escória no Controle de Inclusões}

Após a obtenção da tabela 1, será feita uma análise dos objetivos de cada artigo selecionado em forma de tabela, a tabela 2 , a fim de verificar a influência que importantes parâmetros da escória exercem sobre o controle das inclusões.

$\mathrm{Da}$ análise detalhada da tabela 2 , espera-se que sejam obtidas informações que relacionem parâmetros como basicidade binária, teor de $\mathrm{Al}_{2} \mathrm{O}_{3}$, teor de $\mathrm{SiO}_{2}$ e teor de $\mathrm{MgO}$ com as principais inclusões presentes no refino secundário de aços especiais. Entre os parâmetros que poderão ser analisados nos experimentos futuros estão a população de inclusões, viscosidade efetiva da escória, teor de oxigênio total, composição e formato de inclusões ao longo do tempo. Após a realização dos experimentos, devem ser feitas comparações com os trabalhos revisados a fim de verificar se foi possível chegar nos mesmos resultados e conclusões. Assim, esperase confirmar se o arranjo experimental escolhido está correto para os estudos de inclusões.

\section{RESULTADOS E DISCUSSÃO}

\subsection{Parâmetros Para os Experimentos}

Após a realização dos passos citados anteriormente, foram selecionados 14 trabalhos. Eles estão apresentados em ordem crescente do ano de publicação. Na tabela 1, são apresentados os seguintes parâmetros: tipo de forno, gás de proteção, tipo de cadinho e suas dimensões, massa de aço, massa de escória, momento de adição da escória, proporção escória/aço, temperatura, número de experimentos, tempo e tipo de resfriamento. Os parâmetros que podem ser variados dependo do objetivo de estudo são: massa de escória, proporção escória/aço, número de experimentos e tempo para retirada de amostra. Todos os outros permaneceram constantes. Alguns parâmetros não foram informados pelos autores, assim alguns campos da tabela foram mantidos em branco.

Na sequência, serão analisados e apresentados os parâmetros experimentais citados na tabela 1 a fim de compor o arranjo experimental para estudos posteriores de refino secundário em forno de laboratório. 
Tabela 1. Revisão dos parâmetros de experimentos desenvolvidos em laboratórios

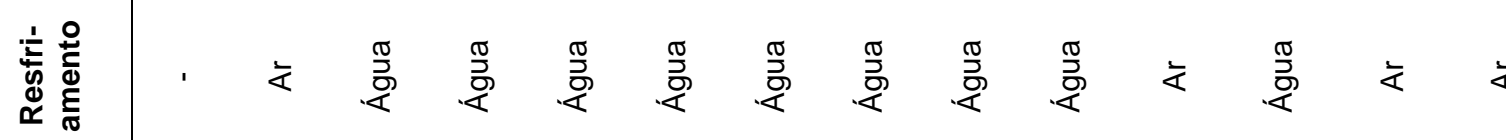

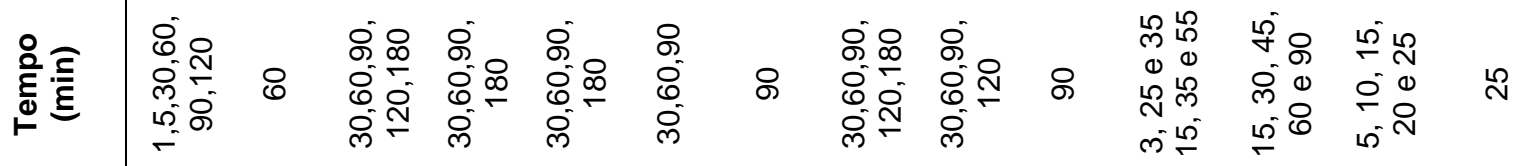

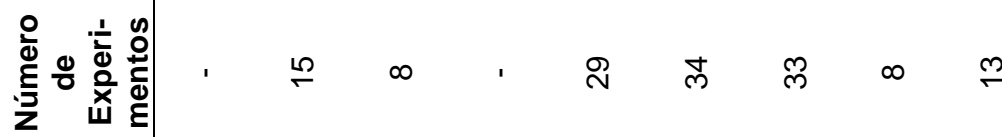

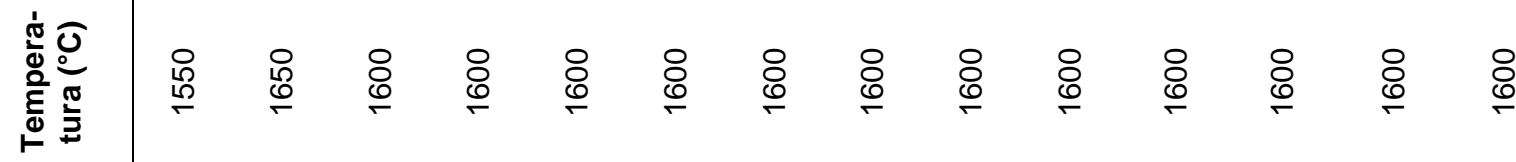

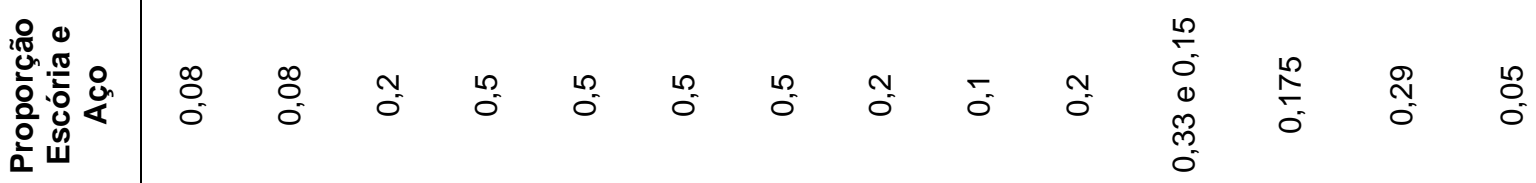

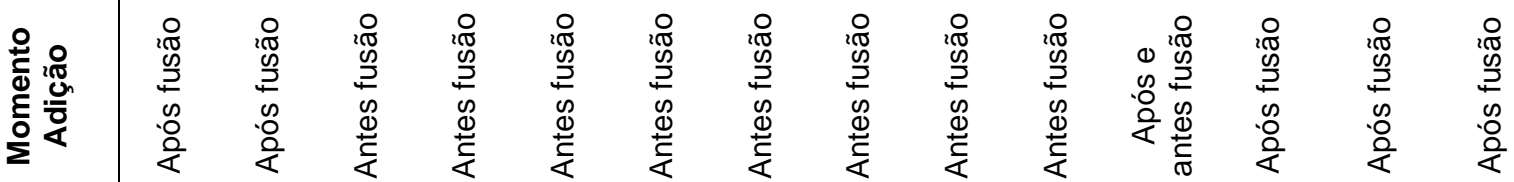

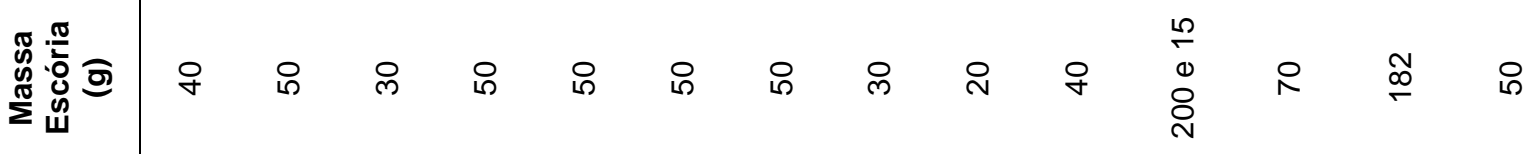

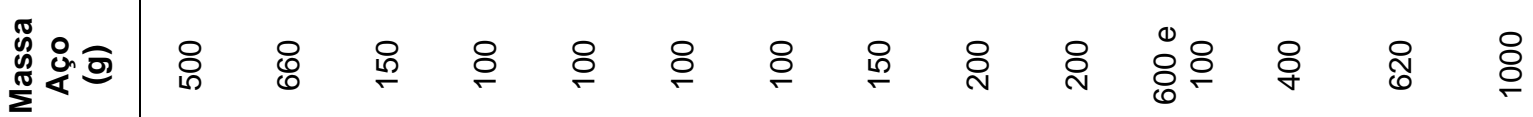

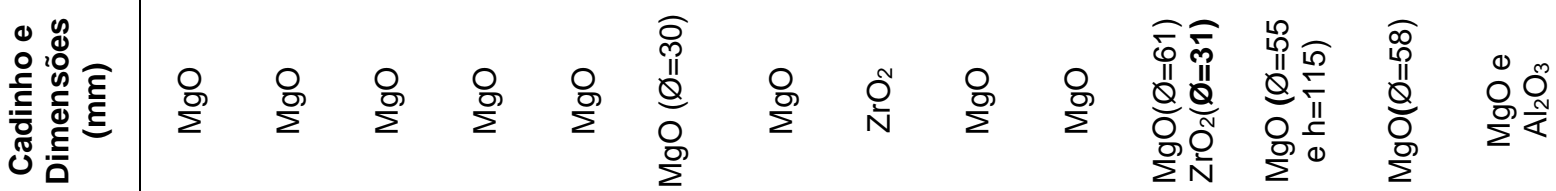

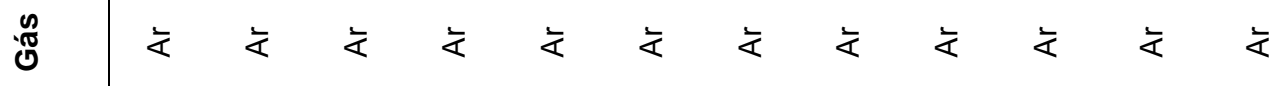

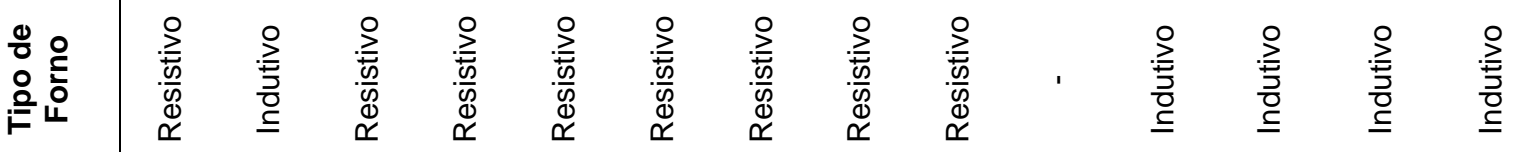

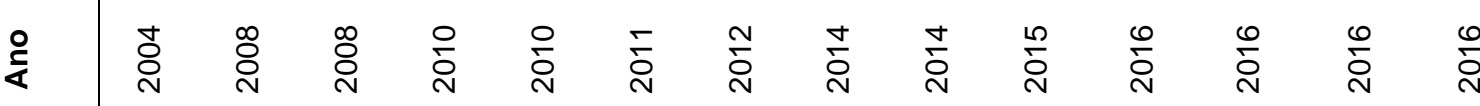

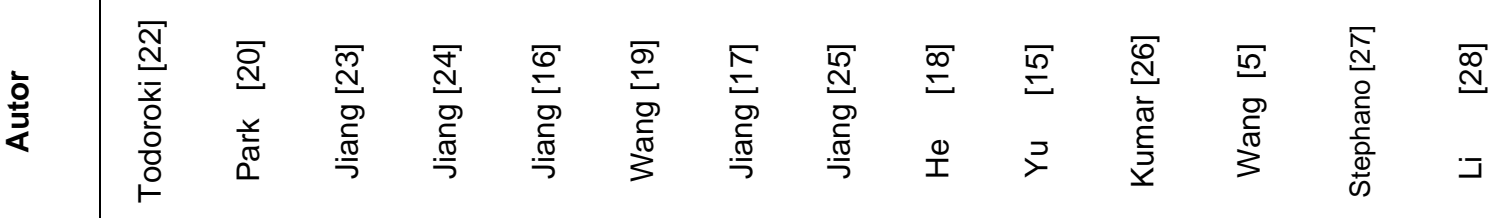

\subsubsection{Tipo de Forno e Gás Inerte}


Os experimentos foram realizados em dois tipos de fornos: indutivo ou resistivo.

A utilização de fornos de indução se caracteriza por utilizar massas de aço e escória mais elevadas quando comparadas ao forno resistivo. Os experimentos em fornos de indução têm a vantagem da existência de linhas de indução que tendem a causar uma agitação natural no banho líquido [22,29].

O princípio de aquecimento de fornos resistivos é a passagem de corrente elétrica através de um condutor elétrico, gerando calor ao equipamento. Devido a passagem de corrente elétrica, pode ser possível a geração de campos magnéticos, capazes de realizar uma pequena agitação no banho líquido [29].

Definiu-se que os experimentos serão realizados em um forno resistivo a alta temperatura.

A escolha foi feita por ser o tipo de forno em operação no LaSid-UFRGS.

O gás inerte de alta pureza empregado será o argônio, seguindo o padrão de todos os experimentos revisados. A função do gás inerte será de proteger o interior do forno e diminuir a pressão parcial de $\mathrm{O}_{2}$, a fim de se evitar possíveis eventos de reoxidação [30]. A pressão parcial no interior do forno foi estimada supondo passagem de gás argônio com 99,999\% de pureza e 1 ppm de $\mathrm{O}_{2}$ em um volume de 4,4 L. Pela equação

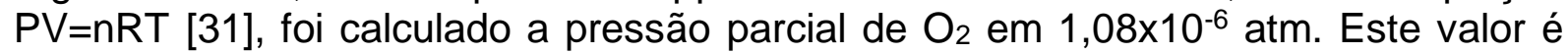
próximo ao encontrado em outro trabalho que utilizou sensores para realizar essa medição [30].

\subsubsection{Tipo de Cadinho, Dimensões do Cadinho, Massa de Aço, Massa de Escória, Momento de Adição e Proporção entre Escória e Aço}

O tipo de cadinho pode variar dependendo do experimento realizado. Cadinhos de $\mathrm{MgO}$ são os mais indicados, pois é semelhante ao tipo de refratário utilizado na maioria das panelas de refino secundário. Cadinhos de $\mathrm{Al}_{2} \mathrm{O}_{3}$ podem ser utilizados desde que seja dada atenção a saturação do $\mathrm{MgO}$ na escória. Em experimentos com este tipo de cadinho, o ideal é que se utilize uma composição química de forma a saturar a escória em $\mathrm{MgO}$. Caso contrário, a formação de inclusões de $\mathrm{MgO}-\mathrm{Al}_{2} \mathrm{O}_{3}$ pode não ocorrer como esperado [28].

Definiu-se que os ensaios serão realizados em cadinhos de $\mathrm{Al}_{2} \mathrm{O}_{3}$ ou $\mathrm{MgO}$, atentando ao ponto de saturação do $\mathrm{MgO}$ quando for utilizado o cadinho de $\mathrm{Al}_{2} \mathrm{O}_{3}$. $\mathrm{O}$ cadinho preferencial para a realização dos experimentos será o de $\mathrm{MgO}$. Os cadinhos utilizados terão diâmetro interno de $20 \mathrm{~mm}$ e altura de $50 \mathrm{~mm}$. Este tamanho permite a colocação de 8 cadinhos dentro do forno em cada experimento. Será utilizado um cadinho protetor de grafita, com diâmetro interno de $100 \mathrm{~mm}$ que será responsável por proteger as amostras e o interior do forno. Esta prática é utilizada em alguns trabalhos apresentados na tabela 1 [26,27]. A massa de aço será de $50 \mathrm{~g}$. A massa de escória poderá variar entre 5 e 12,5 g respeitando os limites de altura do cadinho e será adicionada no início do experimento. A variação da massa de escória, permite o estudo da proporção escória/aço e seu efeito nas inclusões. Quando não for de interesse estudar este fator, será utilizado $10 \mathrm{~g}$ de escória nos experimentos, assim a proporção escória/aço será de 0,2. Esta proporção foi utilizada em alguns trabalhos revisados $[5,15,23,25,26]$.

\subsubsection{Temperatura, Número de Experimentos, Tempo e Resfriamento.}


A temperatura escolhida para os experimentos foi de $1600^{\circ} \mathrm{C}$. O número de experimentos variou de 2 a 33 nas referências citadas na tabela 1, sendo que em alguns não é informado este valor. Os ensaios irão seguir modelos de projetos de experimentos estatísticos. Entre as possibilidades está a realização de projeto fatorial $2^{\mathrm{k}}$ para o estudo das escórias de refino secundário na limpeza dos aços. Com base na revisão, é possível escolher os fatores e os níveis que poderão ser estudados.

O tempo de permanência nesta temperatura será de 90 min. Este tempo já foi estudado, e se chegou neste valor para que o equilíbrio entre aço e escória seja atingido em fornos resistivos [23]. Estudos com diferentes tempos também podem ser realizados, assim é possível analisar a evolução do formato ou composição das inclusões. Neste caso, é proposto retirar amostras em tempos de 30, 60, 90 e 120 minutos. Todas as amostras serão resfriadas em água, seguindo o padrão que é adotado na quase totalidade das referências analisadas.

\subsubsection{Arranjo Experimental}

A partir das observações e definições realizadas nos itens anteriores, os experimentos terão um arranjo experimental conforme ilustra a figura 2.
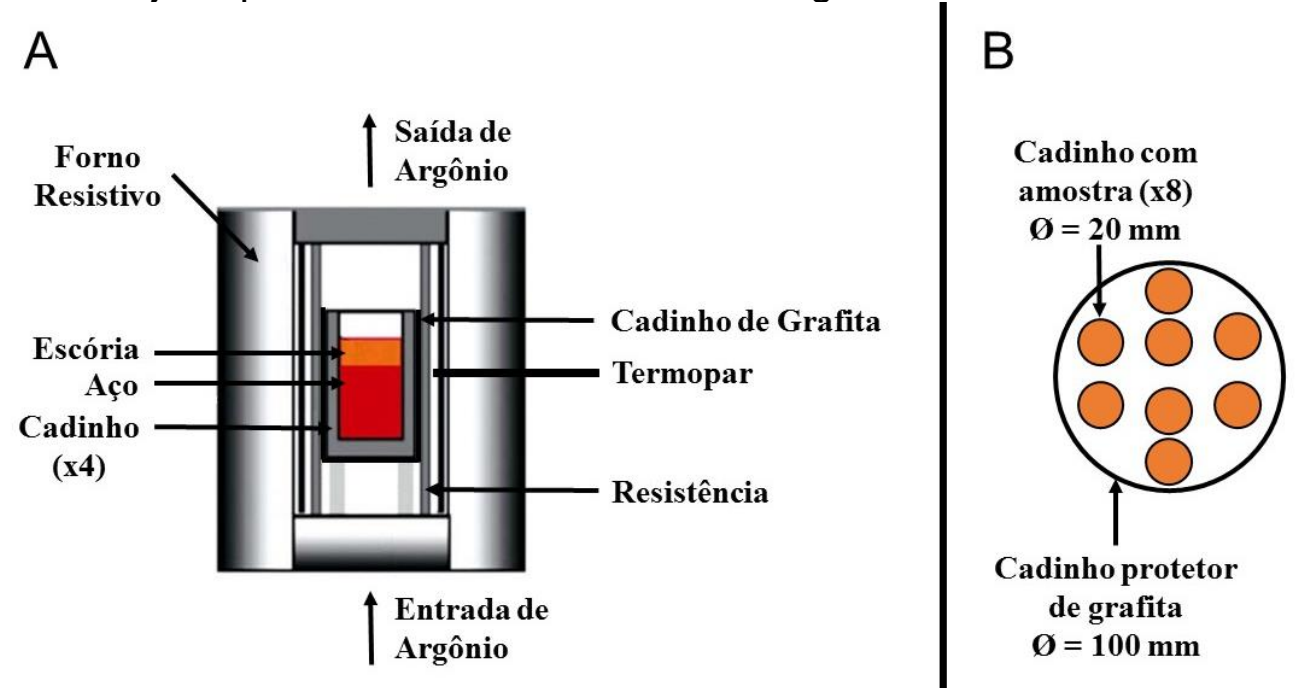

Figura 2. Montagem de experimentos com aço e escória (a) arranjo do forno; (b) arranjo dos cadinhos. Adaptado de He [18]

Será utilizado um forno resistivo, com entrada e saída de gás de alta pureza. Devido as dimensões do cadinho será possível colocar oito cadinhos dentro do forno. Estes cadinhos serão colocados em um cadinho protetor de grafita. $\mathrm{Na}$ temperatura de $1600^{\circ} \mathrm{C}$ irá existir aço e escória líquida dentro do cadinho, caso aconteça algum acidente, o cadinho protetor irá prevenir danos ao interior do forno. O experimento ainda apresenta um termopar para medir a temperatura no interior do forno. $O$ equipamento conta com um sistema de circulação de água para o resfriamento do mesmo, este sistema não está representado na figura 2.

\subsection{Estudos de Escórias de Refino Secundário}

A tabela 2 apresenta o principal objetivo de cada trabalho revisado. Alguns estudos foram selecionados para serem descritos mais detalhadamente. Com essa descrição, será possível analisar o efeito que algumas propriedades da escória podem ter no controle de inclusões não-metálicas. 
Tabela 2. Detalhamento de experimentos desenvolvidos com escórias para o estudo de inclusões

\begin{tabular}{ccl}
\hline Autor & Ano & \\
\hline Todoroki & 2004 & Efeito da sílica sobre as inclusões. \\
[22] & 2008 & Formação de inclusões espinélio pela quantidade de $\mathrm{Al}_{2} \mathrm{O}_{3}$ nas inclusões. \\
Park [20] & 2008 & Formação inclusões $\mathrm{MgO}-\mathrm{Al}_{2} \mathrm{O}_{3}$ variando escória. \\
Jiang [23] & 2008 de Experimento \\
Jiang [24] & 2010 & Evolução do formato das inclusões em escória de alta basicidade. \\
Jiang [16] & 2010 & Controle formato das inclusões ao longo do tempo variando teor $\mathrm{Al}_{2} \mathrm{O}_{3}$. \\
Wang [19] & 2011 & Formação de inclusões em escória alta basicidade e alto $\mathrm{Al}_{2} \mathrm{O}_{3}$. \\
Jiang [17] & 2012 & Formação e remoção de inclusões utilizando escórias alta e baixa basicidade. \\
Jiang [25] & 2014 & Formação de inclusões de baixo ponto de fusão com escória alta basicidade. \\
$\mathrm{He}[18]$ & 2014 & Formação inclusões deformáveis com $\mathrm{SiO}_{2}$ e MnO. \\
Yu[15] & 2015 & Efeito basicidade em inclusões baixo ponto de fusão. \\
Kumar & 2016 & Transferência de cálcio da escória para o aço. \\
[26] & 2016 & Efeito da $\mathrm{Al}_{2} \mathrm{O}_{3}$ e basicidade na composição das inclusões de $\mathrm{Al}_{2} \mathrm{O}_{3}-\mathrm{SiO}_{2}$. \\
Wang [5] & 2016 & \\
Stephano & 2016 & Efeito da composição da escória na composição das inclusões com o tempo. \\
[27] & 2016 & Dois tipos de cadinho para comparar efeito do refratário nas inclusões. \\
Li [28] & 2016 &
\end{tabular}

Todoroki et al. [22] realizaram experimentos para determinar o efeito da sílica na formação de espinélios de $\mathrm{MgO}-\mathrm{Al}_{2} \mathrm{O}_{3}$ em aços inoxidáveis austeníticos. Foram fundidos $500 \mathrm{~g}$ de aço em cadinho de $\mathrm{MgO}$ à $1550^{\circ} \mathrm{C}$ em um forno resistivo com passagem de gás argônio. Após a fusão, foram adicionados alumínio e $40 \mathrm{~g}$ de fluxantes no banho líquido, a escória utilizada possuía 10,6\% em massa de $\mathrm{SiO}_{2}$ em sua composição. Os resultados foram comparados com outro estudo dos mesmos autores em um experimento com escória sem sílica [32]. Após a adição de alumínio e fluxantes, retirou-se amostras em tempos de 1, 5, 30, 60, 90 e 120 minutos. As inclusões foram analisadas via Microscopia Eletrônica de Varredura equipada com Espectroscopia por Dispersão de Energia (MEV/EDS).

Após 30 minutos, os dois experimentos apresentavam inclusões de espinélio de MgO$\mathrm{Al}_{2} \mathrm{O}_{3}$ e, após 90 minutos, somente o experimento com sílica na escória ainda apresentava este tipo de inclusões. O experimento sem sílica apresentava inclusões de $\mathrm{CaO}-\mathrm{Al}_{2} \mathrm{O}_{3}-\mathrm{MgO}$. A sílica se mostrou responsável por controlar o aumento da quantidade de $\mathrm{MgO}$ nas inclusões, mantendo as inclusões na forma de espinélio. Jiang et al. [23] pesquisaram a formação de inclusões de $\mathrm{MgO}-\mathrm{Al}_{2} \mathrm{O}_{3}$ no refino de aços de alta resistência e baixa liga (42CrMo) com escória no sistema $\mathrm{CaO}-\mathrm{SiO}_{2}-\mathrm{Al}_{2} \mathrm{O}_{3}-$ $\mathrm{MgO}$. Neste experimento foram fundidos $150 \mathrm{~g}$ de aço e $30 \mathrm{~g}$ de escória em um cadinho de $\mathrm{MgO}$ na temperatura de $1600^{\circ} \mathrm{C}$. Foi utilizado um forno resistivo com passagem de gás argônio. Primeiramente, foi realizado um experimento para determinar o tempo de equilíbrio entre o metal e a escória. Com base nos resultados, foi estabelecido 90 minutos como o tempo de equilíbrio. O próximo passo foi a realização de sete experimentos com diferentes composições de escória para analisar a formação de inclusões constituídas de $\mathrm{MgO}-\mathrm{Al}_{2} \mathrm{O}_{3}$. As escórias estudadas apresentaram variação na basicidade binária, teor de $\mathrm{Al}_{2} \mathrm{O}_{3}$ e teor de $\mathrm{MgO}$. Todas as amostras foram retiradas e resfriadas em água. As inclusões foram analisadas via MEV/EDS.

Os resultados mostraram que o aumento de $\mathrm{Mg}$ no banho líquido levou ao aumento da quantidade de $\mathrm{MgO}$ nas inclusões. Esse aumento levou a transformação das inclusões de espinélio de globulares para angulares. Foi identificado que se houver cálcio disponível é possível formar inclusões globulares novamente mesmo com o 
aumento de $\mathrm{Mg}$ no banho líquido. Assim como Todoroki [22], também foi possível mostrar o efeito da sílica em estabilizar a existência de inclusões de espinélio.

Jiang et al. [16] estudaram o efeito de escórias com alta basicidade e alta alumina na formação e modificação de inclusões. Foram utilizadas duas escórias (A e B) com basicidade 7, variando o teor de alumina. A escória A apresentou $40 \%$ em massa de alumina enquanto que a escória $B$ apresentou $30 \%$. Foi utilizado um forno resistivo com passagem de gás argônio. Foram fundidos $100 \mathrm{~g}$ de aço, $50 \mathrm{~g}$ de escória e ferroliga de alumínio em cadinho de $\mathrm{MgO}$ a uma temperatura de $1600^{\circ} \mathrm{C}$. Foram retiradas amostras em tempos de 30, 60, 90 e 180 minutos, totalizando 29 amostras. As mesmas foram resfriadas em água e as inclusões foram analisadas via MEV/EDS. A tabela 3 apresenta a evolução no formato das inclusões.

Tabela 3. Evolução do formato das inclusões. Adaptado de Jiang [16]

\begin{tabular}{|c|c|c|c|c|}
\hline $\begin{array}{c}\text { Espécie } \\
\text { Inclusionária }\end{array}$ & $30 \mathrm{~min}$ & $60 \mathrm{~min}$ & $90 \mathrm{~min}$ & $180 \mathrm{~min}$ \\
\hline $\mathrm{MgO}-\mathrm{Al}_{2} \mathrm{O}_{3}$ & & & - & - \\
\hline $\mathrm{MgO}$ & & & & - \\
\hline $\mathrm{CaO}-\mathrm{MgO}-\mathrm{A}_{2} \mathrm{O}_{3}$ & & & & \\
\hline
\end{tabular}

Nas duas escórias foram encontradas inclusões similares dos sistemas $\mathrm{CaO}-\mathrm{MgO}$ $\mathrm{Al}_{2} \mathrm{O}_{3}$ e $\mathrm{MgO}-\mathrm{Al}_{2} \mathrm{O}_{3}$. A presença de cálcio ajudou a transformar as inclusões de formato retangular para esférica. Inclusões de $\mathrm{MgO}-\mathrm{Al}_{2} \mathrm{O}_{3}$ e $\mathrm{MgO}$ formaram uma camada de $\mathrm{CaO}-\mathrm{MgO}-\mathrm{Al}_{2} \mathrm{O}_{3}$ que acabou por torná-las esféricas. A quantidade de $\mathrm{CaO}$ nas inclusões aumentou ao longo do tempo, ao passo que o $\mathrm{MgO}$ diminuiu e o $\mathrm{Al}_{2} \mathrm{O}_{3}$ se manteve praticamente constante. Após 90 minutos, as inclusões eram em sua maioria de $\mathrm{CaO}-\mathrm{MgO}-\mathrm{Al}_{2} \mathrm{O}_{3}$ esféricas e de baixo ponto de fusão. O efeito da alumina interferiu na quantidade de inclusões de MgO. A escória $\mathrm{B}$, com menos alumina, mostrou um número maior de inclusões deste tipo. Esse efeito se deu devido a escória $B$ apresentar $\mathrm{MgO}$ saturado na escória, assim a atividade do Mg era maior, propiciando a formação de inclusões de MgO.

Wang et al. [19] realizaram ensaios em laboratório e na indústria nas mesmas condições que Jiang [16]: alta basicidade e variação de alumina. Foram obtidos resultados semelhantes, ao aumentar a basicidade de 5 para 7 e a quantidade de alumina de 20 para $40 \%$ em massa. A porcentagem de inclusões do sistema $\mathrm{CaO}$ $\mathrm{MgO}-\mathrm{Al}_{2} \mathrm{O}_{3}$ de baixo ponto de fusão subiu de 24,4 para $81,7 \%$.

Jiang et al. [17] estudaram o efeito da basicidade binária e da alumina para atingir uma boa limpeza e inclusões de baixo ponto de fusão. Foram estudadas três escórias: $\mathrm{A}\left(\mathrm{B}_{2}=7\right.$ e $\left.\mathrm{Al}_{2} \mathrm{O}_{3}=40 \%\right), \mathrm{B}\left(\mathrm{B}_{2}=7\right.$ e $\left.\mathrm{Al}_{2} \mathrm{O}_{3}=30 \%\right)$ e $\mathrm{C}\left(\mathrm{B}_{2}=3,5\right.$ e $\left.\mathrm{Al}_{2} \mathrm{O}_{3}=25 \%\right)$. Durante $\mathrm{o}$ experimento foram fundidos $100 \mathrm{~g}$ de aço e $50 \mathrm{~g}$ de escória na temperatura de $1600^{\circ} \mathrm{C}$ em cadinho de $\mathrm{MgO}$. Foi utilizado um forno resistivo com passagem de gás argônio. As amostras foram mantidas por 90 minutos na temperatura indicada e resfriadas em água. A população de inclusões foi analisada por MEV/EDS.

As inclusões presentes nos experimentos com escória $A$ e $B$ apresentaram formato esférico de acordo com estudos anteriores [16,19]. Com a escória $A$, as inclusões eram em sua maioria de baixo ponto de fusão, enquanto na escória B houve maior número de inclusões de $\mathrm{MgO}$. Isso ocorreu devido a maior quantidade de alumina da escória $A$, diminuindo o ponto de fusão das inclusões, e pelo fato da escória $B$ apresentar saturação do MgO na escória, aumentando a atividade do Mg. A escória 
C apresentou grande número de inclusões com formato irregular e distantes da zona de baixo ponto de fusão.

O autor se utiliza da figura 3, apresentada por Valdez [14] para verificar a capacidade de cada escória em dissolver inclusões de $\mathrm{Al}_{2} \mathrm{O}_{3}$ e espinélios.

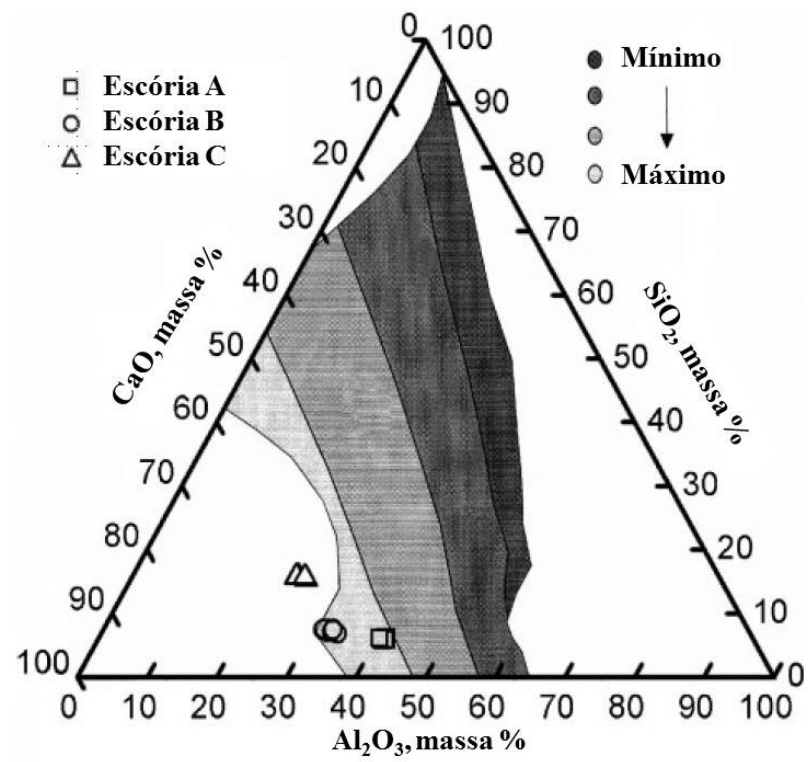

Figura 3. Capacidade de escórias para a dissolução de inclusões. Adaptado de Jiang [17]

A figura 3 apresenta regiões do diagrama ternário $\mathrm{CaO}-\mathrm{MgO}-\mathrm{Al}_{2} \mathrm{O}_{3}$ e compara a capacidade de remover inclusões da escória, a partir da sua composição química [14]. As escórias A e B se encontram na mesma região e apresentam forte capacidade de dissolução. A escória $\mathrm{C}$ se encontra fora da região líquida a $1600^{\circ} \mathrm{C}$ e não é favorável para dissolução.

Yu et al. [15] pesquisaram o efeito de escórias com basicidade binária entre 3,5 e 5 sobre a formação de inclusões de baixo ponto de fusão. Foram realizados testes em forno de laboratório e na indústria. Os experimentos foram realizados em um forno resistivo com passagem de gás argônio. Foram fundidos $200 \mathrm{~g}$ de aço e $40 \mathrm{~g}$ de escória em cadinho de $\mathrm{MgO}$ na temperatura de $1600^{\circ} \mathrm{C}$. As amostras foram mantidas nesta temperatura por 90 minutos para atingir o equilíbrio entre aço e escória, então as mesmas foram resfriadas em água. Para análise de inclusões aplicou-se a técnica de MEV/EDS. Os resultados mostraram que o uso de escória com basicidade 4,5 e $20 \%$ em massa de $\mathrm{Al}_{2} \mathrm{O}_{3}$ foi eficiente em manter inclusões de alta temperatura de fusão do sistema $\mathrm{CaO}-\mathrm{MgO}-\mathrm{Al}_{2} \mathrm{O}_{3}$.

Kumar et al. [26] estudaram os mecanismos sobre a transferência de cálcio da escória para o aço, e do aço para a inclusão. Foram feitos experimentos em dois tipos de cadinho, $\mathrm{MgO}$ e $\mathrm{ZrO}_{2}$. Nos experimentos em cadinho de $\mathrm{MgO}$ a adição da escória foi realizada após a fusão do aço, ao passo que no de $\mathrm{ZrO}_{2}$, a adição foi feita antes da fusão do aço. O experimento realizado com adição antes da fusão apresentou um menor valor no teor de oxigênio total. Isso se deu devido ao fato da escória fundir antes do aço, cobrindo partes do aço sólido e assim ocorrendo uma melhor limpeza inclusionária. Os resultados sobre a transferência de cálcio mostraram uma competição entre a redução de $\mathrm{CaO}$ e $\mathrm{MgO}$. Sendo a redução de $\mathrm{MgO}$ mais favorável em alguns casos, com isso, diminuindo a quantidade de cálcio no banho líquido reduzido a partir da escória.

Wang et al. [5] estudaram o efeito da alumina e da basicidade binária na limpeza de aços inoxidáveis. Foi utilizado um forno de indução com passagem de gás argônio 
para fundir $400 \mathrm{~g}$ de aço em um cadinho de $\mathrm{MgO}$ na temperatura de $1600^{\circ} \mathrm{C}$. Após a fusão foi adicionado $70 \mathrm{~g}$ de escória e foram retiradas amostras após 15, 30, 45, 60 e 90 minutos. As amostras foram resfriadas em água e as inclusões analisadas via MEV/EDS.

Os resultados mostraram que, em basicidade maiores que 2,5, a transição para inclusões de baixo ponto de fusão do sistema $\mathrm{CaO}-\mathrm{Al}_{2} \mathrm{O}_{3}-\mathrm{SiO}_{2}-\mathrm{MgO}$ foi acelerada. As escórias de alta basicidade apresentaram um bom poder desoxidante e boa capacidade para absorver inclusões. Com o aumento da basicidade, houve aumento de alumínio dissolvido do aço líquido, favorecendo a formação de inclusões de MgO$\mathrm{Al}_{2} \mathrm{O}_{3}$. Em níveis iguais de basicidade e variando o teor de alumina, as inclusões evoluíram para o sistema $\mathrm{CaO}-\mathrm{Al}_{2} \mathrm{O}_{3}-\mathrm{SiO}_{2}-\mathrm{MgO}$ ao invés de espinélios no experimento com maior teor de alumina. Quando o conteúdo de alumina foi maior que $21 \% \mathrm{em}$ massa na composição da escória, houve um aumento no teor de oxigênio total, além de um efeito negativo na capacidade de absorver inclusões.

Li et al. [28] investigaram a formação e evolução de inclusões em aços especiais com baixo teor de carbono, retirando amostras desde a etapa de refino secundário até o lingotamento contínuo. Um dos fatores estudados foi a influência do material refratário da panela. Para isso foram realizados testes em laboratório utilizando cadinhos de $\mathrm{MgO}$ e de $\mathrm{Al}_{2} \mathrm{O}_{3}$ para avaliar o impacto do refratário na formação das inclusões.

Foram fundidos $1000 \mathrm{~g}$ de aço em um forno de indução a vácuo com passagem de gás com alta pureza. Foi estabelecida a temperatura de $1600^{\circ} \mathrm{C}$, após a fusão do aço foram adicionados alumina e $50 \mathrm{~g}$ de escória. Em um dos experimentos com cadinho de $\mathrm{MgO}$ não foi adicionado escória. Após 25 minutos o aço líquido foi solidificado em moldes de ferro fundido, produzindo pequenos lingotes usados posteriormente para análise de inclusões via MEV/EDS.

Os resultados mostraram que após 25 minutos, o experimento no cadinho de $\mathrm{Al}_{2} \mathrm{O}_{3}$ apresentava inclusões de $\mathrm{CaO}-\mathrm{Al}_{2} \mathrm{O}_{3}$. Neste mesmo tempo, com o cadinho de $\mathrm{MgO}$, foram formadas inclusões de $\mathrm{MgO}-\mathrm{Al}_{2} \mathrm{O}_{3}$ mesmo sem a presença de escória. Isso mostrou que o alumínio dissolvido no aço líquido reage com o material refratário, tendo influência na formação de inclusões de $\mathrm{MgO}-\mathrm{Al}_{2} \mathrm{O}_{3}$. Ao aumentar a atividade do $\mathrm{Mg}$ no experimento utilizando cadinho de $\mathrm{Al}_{2} \mathrm{O}_{3}$, inclusões de $\mathrm{MgO}-\mathrm{Al}_{2} \mathrm{O}_{3}$ foram formadas. Entre os trabalhos revisados (tabela 1 ) são citados dois tipos de escória que são usualmente aplicados durante o processo de refino secundário utilizando o sistema de escórias $\mathrm{CaO}-\mathrm{SiO}_{2}-\mathrm{Al}_{2} \mathrm{O}_{3}-\mathrm{MgO}$. Escórias com baixa basicidade, menor do que $2 \mathrm{e}$ baixa concentração de $\mathrm{Al}_{2} \mathrm{O}_{3}$, que apresentam inclusões de baixo ponto de fusão. Escórias com alta basicidade, entre 5 e 7, com aproximadamente $20 \%$ em massa de $\mathrm{Al}_{2} \mathrm{O}_{3}$, apresentam inclusões de alto ponto de fusão e maior quantidade de $\mathrm{MgO}$ em sua composição $[15,16,19]$. Entretanto é possível concluir que outros sistemas podem ser utilizados para obter resultados semelhantes. Entre eles a utilização de escórias com alta basicidade (entre 5 e 7 ) e alta alumina ( 40\%) para obtenção de inclusões de baixo ponto de fusão $[16,17,19]$.

\section{CONCLUSÕES}

Com base na revisão de diversos estudos desenvolvidos em escala laboratorial e dos resultados apresentados, pode-se concluir que os parâmetros para realização dos experimentos serão:

- Forno: Resistivo;

- Gás de proteção: Argônio;

- Material do cadinho: MgO; 
- Número cadinhos por experimento: 8 cadinhos;

- Massa de aço: $50 \mathrm{~g}$;

- Massa de escória e momento de adição: 10 g, antes da fusão;

- Proporção escória/aço: 0,2;

- Temperatura: $1600^{\circ} \mathrm{C}$;

- Número de amostras: escolhido a partir de fatorial $2^{\mathrm{k}}$;

- Tempo: 90 minutos;

- Resfriamento: Água.

A partir da descrição dos experimentos selecionados, pode-se concluir, com relação aos efeitos das escórias no controle de inclusões, o seguinte:

- Basicidade Binária:

$\checkmark$ Aumento entre 3-4 para 6-8, melhorou a capacidade de dissolução de inclusões de espinélio e de $\mathrm{Al}_{2} \mathrm{O}_{3}$ [17].

$\checkmark$ Aumento de 3,5 para 4,5 , junto a $20 \%$ em massa de $\mathrm{Al}_{2} \mathrm{O}_{3}$, aumentou o ponto de fusão das inclusões [15].

$\checkmark$ Aumento até 4 diminui o teor de oxigênio total, acima de 4 se manteve estável [5].

- Teor de Alumina:

$\checkmark$ Aumento da atividade da alumina, elevou a quantidade de $\mathrm{Al}_{2} \mathrm{O}_{3}$ na composição

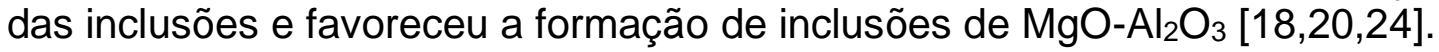

$\checkmark$ Aumento para $40 \%$ em massa, junto a basicidade 7 , aumentou a quantidade de inclusões de baixo ponto de fusão $[16,17,19]$.

$\checkmark$ Aumento para valores acima de $21 \%$ em massa, junto a basicidade 2,5 , elevou o teor de oxigênio total [5].

- Teor de Sílica:

$\checkmark$ Aumento de 0 a $20 \%$ em massa, elevou o número de inclusões de $\mathrm{MgO}-\mathrm{Al}_{2} \mathrm{O}_{3}$ $[22,23,32]$.

- Teor de MgO:

$\checkmark$ Ao atingir o ponto de saturação de $\mathrm{MgO}$ na escória, a formação de inclusões de $\mathrm{MgO}-\mathrm{Al}_{2} \mathrm{O}_{3}$ é favorecida $[16,17]$.

\section{Agradecimentos}

Pedro Alves agradece aos professores Wagner Bielefeldt e Antônio Vilela pela orientação e apoio, aos colegas do LASID, principalmente aos que ajudaram diretamente no desenvolvimento deste trabalho, e a Fundação Luiz Englert pela bolsa de estudos.

\section{REFERÊNCIAS}

1 Mourão MB. Introdução à Siderurgia. São Paulo: ABM; 2007.

2 Reis BH, Bielefeldt WV, Vilela ACF. Efficiency of Inclusion Absorption by Slags during Secondary Refining of Steel. ISIJ International. 2014;54(7):1584-1591.

3 Rocha VC, Pereira JAM, Yoshioka A, Bielefeldt WV, Vilela ACF. Evaluation of Secondary Steelmaking Slags and Their Relation with Steel Cleanliness. Metallurgical and Materials Transactions B: Process Metallurgy and Materials Processing Science. 2017;48(June):1423-1432.

4 Fruehan RJ. Unique functions of slags in steelmaking. 7th International Conference on Molten Slags, Fluxes, and Salts. 2004. 263-270.

5 Wang Q, Wang L, Chou K. Effect of $\mathrm{Al}_{2} \mathrm{O}_{3}$ Content in Top Slag on Cleanness of Stainless Steel Fe-13Cr. 10th International Conference on Molten Slags, Fluxes, and Salts. 2016. 155-163. 
6 Pretorius EB. The Effect of Fluorspar in Steelmaking Slags. [acesso em 25 maio 2017]. Disponível em: http://etech.Iwbref.com/Downloads/Theory/The\%20Effect\%20of\%20Fluorspar\%20in\%20Steelmaking\%20SI ags.pdf.

7 Suito H, Inoue R. Thermodynamics clean Steels on Control of Inclusions Composition in Ultra. ISIJ International. 1996;36(5):528-536.

8 Yoon B-H, Heo K-H, Kim J-S, Sohn H-S. Improvement of steel cleanliness by controlling slag composition. Ironmaking \& Steelmaking. 2002;29(3):214-217.

9 Pretorius EB, Oltmann HG, Schart BT. An Overview of Steel Cleanliness From an Industry Perspective. AISTech 2013 Proceedings. 2013. 993-1026.

10 Zhang LF, Thomas BG. State of the Art in Evaluation and Control of Steel Cleanliness. ISIJ International. 2003;43(3):271-291.

11 Holappa LEK, Helle AS. Inclusion Control in High-Performance Steels. Journal of Materials Processing Tech. 1995;53(1-2):177-186.

12 Bartosiaki BG, Pereira JAM, Bielefeldt WV, Vilela ACF. Assessment of inclusion analysis via manual and automated SEM and total oxygen content of steel. Journal of Materials Research and Technology. 2015;4(3):235-240.

13 Kiessling R. Non Metallic Inclusions in Steels Part I-IV. London: The Institute of Materials; 1978.

14 Valdez M, Shannon GS, Sridhar S. The Ability of Slags to Absorb Solid Oxide Inclusions. ISIJ International. 2006;46(3):450-457.

15 Yu HX, Wang XH, Zhang J, Wang WJ. Characteristics and Metallurgical Effects of Medium Basicity Refining Slag on Low Melting Temperature Inclusions. Journal of Iron and Steel Research International. 2015;22(7):573-581.

16 Jiang $\mathrm{M}$, Wang $\mathrm{XH}$, Wang WJ. Control of non-metallic inclusions by slag-metal reactions for high strength alloying steels. Steel Research International. 2010;81(9):759-765.

17 Jiang $\mathrm{M}$, Wang $\mathrm{XH}$, Wang WJ. Study on refining slags targeting high cleanliness and lower melting temperature inclusions in Al killed steel. Ironmaking \& Steelmaking. 2012;39(1):20-25.

18 He XF, Wang XH, Chen SH, Jiang M, Huang FX, Wang WJ. Inclusion composition control in tyre cord steel by top slag refining. Ironmaking \& Steelmaking. 2014;41(9):676-684.

19 Wang X, Jiang M, Chen B, Wang W. Study of Non-metallic Inclusions in High Strength Alloy Steel Refined by Using High Basicity and High $\mathrm{Al}_{2} \mathrm{O}_{3}$ Content Slag. Springer-Verlag Berlin Heidelberg and Metallurgical Industry Press. 2011. 485-494.

20 Park $\mathrm{JH}$. Thermodynamic investigation on the formation of inclusions containing $\mathrm{MgAl}_{2} \mathrm{O}_{4}$ spinel during $16 \mathrm{Cr}-14 \mathrm{Ni}$ austenitic stainless steel manufacturing processes. Materials Science and Engineering $\mathrm{A}$. 2008;472(1-2):43-51.

21 Chen SH, Jiang M, He XF, Wang XH. Top slag refining for inclusion composition transform control in tire cord steel. International Journal of Minerals, Metallurgy and Materials. 2012;19(6):490-498.

22 Todoroki H, Mizuno K. Effect of Silica in Slag on Inclusion Compositions in 304 Stainless Steel Deoxidized with Aluminum. ISIJ International. 2004;44(8):1350-1357.

23 Jiang $\mathrm{M}$, Wang $\mathrm{X}$, Chen $\mathrm{B}$, Wang W. Formation of $\mathrm{MgO} \cdot \mathrm{Al}_{2} \mathrm{O}_{3}$ Inclusions in High Strength Alloyed Structural Steel Refined by CaO-SiO2-Al2O3-MgO Slag. ISIJ International. 2008;48(7):885-890.

24 Jiang M, Wang X, Chen B, Wang W. Laboratory Study on Evolution Mechanisms of Non-metallic Inclusions in High Strength Alloyed Steel Refined by High Basicity Slag. ISIJ International. 2010;50(1):95-104.

25 Jiang $\mathrm{M}$, Wang $\mathrm{XH}$, Pak JJ. Formation of low-melting-point inclusions in al-deoxidized steel refined by highbasicity calcium aluminate slag in $\mathrm{ZrO}_{2}$ crucible experiments. Metallurgical and Materials Transactions $\mathrm{B}$ : Process Metallurgy and Materials Processing Science. 2014;45(4):1248-1259.

26 Kumar D, Pistorius PC. A Study on Calcium Transfer From Slag To Steel and Its Effect on Modification of Alumina and Spinel Inclusions. 10th International Conference on Molten Slags, Fluxes, and Salts. 2016. 145-154.

27 Piva SPT, Pistorius PC. Effect of Ladle Furnace Slag Composition in Si-Mn Killed. 10th International Conference on Molten Slags, Fluxes, and Salts. 2016. 117-126.

28 Li JZ, Jiang M, He XF, Sun W, Wang XH. Investigation on Nonmetallic Inclusions in Ultra-Low-Oxygen Special Steels. Metallurgical and Materials Transactions B: Process Metallurgy and Materials Processing Science. 2016;47(4):2386-2399.

29 Grzella J, Sturm P, Krüger J, Reuter MA, Carina K, Probst T. Metallurgical Furnaces. Weinheim: WileyVCH; 2008.

30 Yan P, Arnout S, Van Ende MA, Zinngrebe E, Jones T, Blanpain B, et al. Steel Reoxidation by Gunning Mass and Tundish Slag. Metallurgical and Materials Transactions B: Process Metallurgy and Materials Processing Science. 2015;46(3):1242-1251.

31 Poling BE, Prausnitz JM, O'connell JP. The properties of gases and liquids (Vol. 5). New York: Mcgraw-hill; 2001.

32 Todoroki H, Mizuno K. Variation of Inclusion Composition in 304 Stainless Steel Deoxidized with Aluminum. Iron \& steelmaker ISS Transactions. 2003;30(3):60-67. 